\title{
"Obesity-Years" Burden May Predict Reversibility in Heart Failure With Preserved Ejection Fraction
}

\author{
Anand Chockalingam ${ }^{1,2 *}$ \\ ${ }^{1}$ Division of Cardiovascular Medicine, Department of Medicine, Five Hospital Drive, University of Missouri, Columbia, MO, \\ United States, ${ }^{2}$ Home Cardiac Rehabilitation Program, The Harry S. Truman Memorial Veterans' Hospital, Columbia, MO, \\ United States
}

Keywords: obesity cardiac remodeling, heart failure, severe obesity, HFpEF-heart failure with preserved ejection fraction, resilience, obesity cardiomyopathy, reversible cardiomyopathy

\section{INTRODUCTION}

Obesity is increasing in all age groups and understanding its contribution to Heart Failure (HF) is central for optimizing care. Heart Failure with preserved ejection fraction (HFpEF) is a clinical syndrome where symptoms and signs of HF occur due to high ventricular filling pressure despite normal left ventricular ejection fraction. In recent trials, HFpEF accounts for over half of all heart failure diagnoses (1). Through careful hemodynamic and echocardiographic characterization, "obese HFpEF" has been identified as a distinct phenotype within the larger HFpEF population (2). Compared to "typical HFpEF", "obese HFpEF" patients have higher BMI and experience onset

OPEN ACCESS

Edited by:

Kristin Stanford,

The Ohio State University,

United States

Reviewed by:

Micha Tobias Maeder,

Kantonsspital St. Gallen, Switzerland

*Correspondence:

Anand Chockalingam

chockalingama@health.missouri.edu

Specialty section:

This article was submitted to Cardiovascular Epidemiology and

Prevention,

a section of the journal

Frontiers in Cardiovascular Medicine

Received: 25 November 2021

Accepted: 04 January 2022

Published: 07 February 2022

Citation:

Chockalingam A (2022)

"Obesity-Years" Burden May Predict

Reversibility in Heart Failure With

Preserved Ejection Fraction.

Front. Cardiovasc. Med. 9:821829.

doi: 10.3389/fCvm.2022.821829 of clinical decompensation a decade earlier. Recent insights help in better understanding the mechanisms of obesity mediated HFpEF. If recognized early, much of the cardiac pathology of obese HFpEF may be reversible with adequate weight reduction.

\section{OBESITY TRAJECTORY}

Childhood obesity in the US (with onset between 2 and 5 years of age) has dramatically increased from $5 \%$ in 1970 s to $19 \%$ in 2018 . Severe childhood obesity has similarly increased from $1 \%$ in 1970 s to $6 \%$ in 2018 with corresponding increase in lifetime cardiac workload. HF remains a clinical diagnosis despite recent developments in cardiac imaging and biomarkers. The Framingham criteria were validated in systolic HF patients in the 1960s when prevalence of severe obesity was $<1 \%$ of the US population. When decompensated HF despite normal systolic function (HFpEF) was first reported in the 1990s, the prevalence of severe obesity was below $10 \%$ in the US (3). Earlier HFpEF trials excluded obese patients further limiting our understanding of its real contribution to decompensation. With severe obesity in the US projected to exceed $24 \%$ by 2030 , reliable tools are needed to quantify cardiac pathology (4).

\section{OBESITY CARDIOMYOPATHY}

Obesity cardiomyopathy (OC) describes obesity induced increase in total blood volume and cardiac output with resultant left ventricular dilation, increased left ventricular wall stress, compensatory left ventricular hypertrophy, and left ventricular diastolic dysfunction (5).

Extreme obesity with body mass indexes (BMI) of $40-70 \mathrm{~kg} / \mathrm{m}^{2}$ can cause OC in younger adults without hypertension, coronary artery disease, and other comorbidities (5). Systolic dysfunction has been variably recognized in association with severe obesity, although the role of confounding etiologies like hypertension, sleep apnea, atrial fibrillation and sepsis cannot be excluded. Similar to tachycardia, peripartum, inflammatory, and Takotsubo cardiomyopathies, OC is also a reversible 
cardiomyopathy (6). Significant (43 kg) weight loss through gastric bypass in younger adults (30-45 years of age) improves cardiac dimensions, filling pressure and diastolic function, confirming $\mathrm{OC}$ is indeed reversible (7). According to the ACC/AHA stages of HF, structural changes of OC would correspond to stage B HF since clinical decompensation is not yet evident in these patients (8).

\section{“OBESITY-YEARS” BURDEN}

Duration of obesity plays a central role in determining cardiac burden. The probability of OC increased dramatically from $20 \%$ when duration of severe obesity was 15 years to $95 \%$ at 25 years (9). Patients may not reliably recall their weight throughout life. In busy clinical settings, without periodic visits to the same healthcare system, quantifying obesity burden is challenging. Recognizing the numerous caveats, "Obesity-years" may offer an estimate of lifetime burden of obesity. Cohorts of "typical" HFpEF, obese HFpEF and OC are derived from published age (X axis) and BMI (Y axis) standard deviation ranges in Figure 1 $(1,2,5)$. When empirically traced, the intercept of age + BMI $=100$ passes through all these 3 cohorts. Thus, in primary care and HF settings, this may represent an arbitrary threshold of HF

"Obesity - Years" predicts Heart Failure risk in severely obese patients

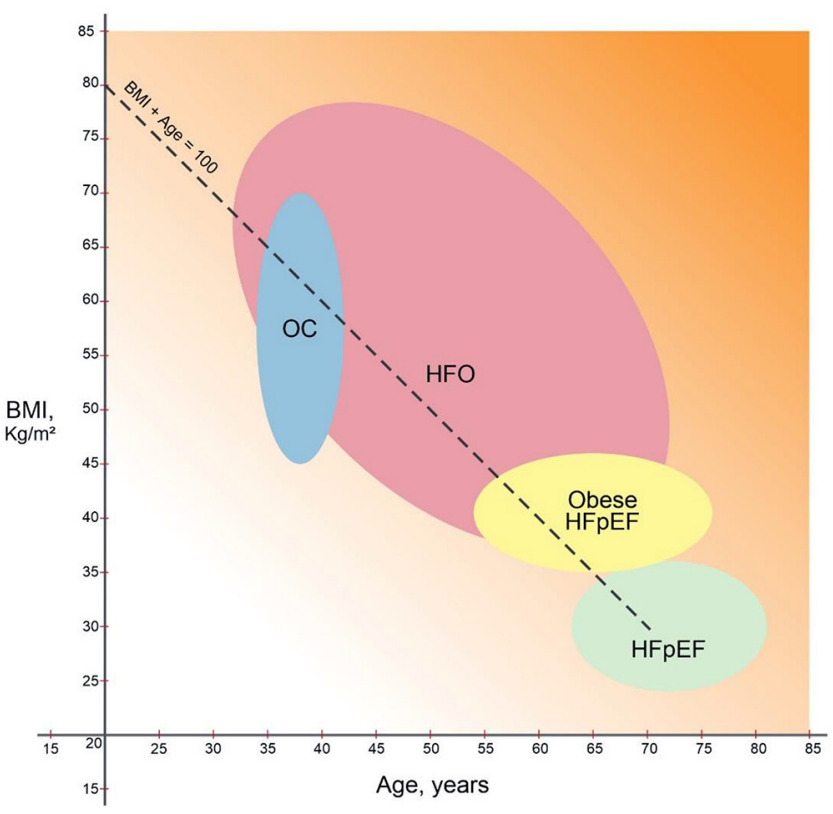

FIGURE 1 | "Obesity-Years" burden and cardiac resilience in heart failure $(1,2,5,12)$. Heart failure with preserved ejection fraction ("typical" HFpEF) (1), obese HFpEF (2), and obesity cardiomyopathy (OC) groups (5) derived from standard deviation ranges for age (in years, $X$-axis) and BMl ( $\mathrm{kg} / \mathrm{m}^{2}, \mathrm{Y}$-axis). Lifetime cumulative risk for HF of obesity (HFO) increases when "obesity-years" (BMI + age) reaches 100. Increasing background gradient denotes declining cardiac resilience with age beyond 70 years. Thus, younger HFO patients can expect resolution of cardiac symptoms and HFpEF reversal with sufficient weight loss (12). risk due to obesity. BMI of $40 \mathrm{~kg} / \mathrm{m}^{2}$ can cause HFpEF by the age of 65 years (2). This hypothetical "obesity-years" threshold (when age + BMI exceeds 100) to predict stage B HFpEF warrants further validation.

\section{HEART FAILURE OF OBESITY}

A new term, heart failure of obesity (HFO), implies obesity can account for HF independent of other comorbidities. HFO would include obese HFpEF patients with symptoms and signs of decompensation (stage C) as well as OC patients with structural cardiac abnormalities and diastolic dysfunction (stage B). Weight reduction may improve symptoms even in instances when HF is primarily due to hypertension, ischemic cardiomyopathy, valve disease or renal failure. HFO is likely by the age of 30 years if BMI exceeds $70 \mathrm{~b} \mathrm{~kg} / \mathrm{m}^{2}$ and when an "asymptomatic" individual with BMI $50 \mathrm{~kg} / \mathrm{m}^{2}$ reaches the age of 50 years.

Clinical diagnosis of $\mathrm{HF}$ is challenging in severe obesity. Clinical and biomarker criteria to diagnose $\mathrm{HF}$ have yet to be validated in the severely obese. HFpEF is shifting to increasingly younger populations. We encounter stage $\mathrm{C}$ decompensated HF in 30-55-year-old patients when the BMI is $60-80 \mathrm{~kg} / \mathrm{m}^{2}$. Typical HF symptoms of dyspnea and edema may be attributed to obesity, deconditioning and arthritis. Brain natriuretic peptide increase is blunted in the obese and thus unreliable in diagnosing HFO (10). Often weight precludes catheterization, and echocardiography images are suboptimal. Stage C clinical decompensated HF is widely considered a maladaptive irreversible chronic disease with adverse prognosis (1). In HFO, however, both stages B and C appear to fall along a continuum of compensatory cardiac adaptations to the excess workload. Likelihood of Stage B HFO increases when age + BMI approaches 100. The diagnosis of Stage C HFO requires pulmonary congestion, volume overload or need for diuretics.

\section{RESILIENCE AND AGING}

Diverse evidence from aging and resilience research, gastric bypass, and calorie restriction literature suggests potential for reversing $\mathrm{OC}$ and obese $\mathrm{HFpEF}$. Broadly, resilience is the ability of a system to maintain specific functions in the face of change. Cardiac resilience denotes the ability to maintain adequate cardiac output despite comorbidities (hypertension, valve disease, or renal dysfunction) and acute stressors (exercise, anemia, surgery, or infection). Resilience declines significantly with aging. Compared to a 30-year-old, the risk of all cause death increases 8 -fold by age 56 and 64 -fold by age 78 . Resilience and biological aging can be improved with lifestyle. In a randomized trial of healthy males, an 8-week diet and lifestyle intervention reversed biological age by 3.2 years (11). Hemodynamic load and inflammation appear to mediate most of the adaptive cardiac changes in HFO. Thus, patients achieving over $20 \%$ weight reduction before age 60-70 years may see complete reversal of HF (12).

Patients with a long history of obesity need to be counseled about the cumulative "obesity-years" burden and may benefit 
from cardiac evaluation. Empagliflozin reduces rehospitalization in HFpEF. ${ }^{1}$ Randomized trial with calorie restriction and exercise derived 7-10\% weight loss benefits obese HFpEF patients (13). Gastric bypass studies have shown diastolic cardiac abnormalities are due to obesity and improve when $>20 \%$ weight loss is achieved (7). The dramatic reductions in symptoms, rehospitalizations, and medication requirements observed in HFO patients who lose $20-35 \%$ of body weight may be due intact resilience pathways (14). Based on patient feedback with holistic mind-body methods, we are building metabolic HF clinics targeting several resilience pathways for sustaining weight loss (12). Intentional 15-25\% weight loss by $65-70$ years in obese symptomatic patients may reverse HFpEF (15).

\section{CONCLUSIONS}

HF of obesity manifests at younger age, with fewer associated comorbidities and carries a better prognosis than other HFpEF

\section{REFERENCES}

1. Anker SD, Butler J, Filippatos G, Ferreira JP, Bocchi E, Böhm M, et al. Empagliflozin in heart failure with a preserved ejection fraction. N Engl J Med. (2021) 385:1451-61. doi: 10.1056/NEJMoa2107038

2. Obokata M, Reddy YNV, Pislaru SV, Melenovsky V, Borlaug BA. Evidence supporting the existence of a distinct obese phenotype of heart failure with preserved ejection fraction. Circulation. (2017) 136:6-19. doi: 10.1161/CIRCULATIONAHA.116.026807

3. Oktay AA, Rich JD, Shah SJ. The emerging epidemic of heart failure with preserved ejection fraction. Curr Heart Fail Rep. (2013) 10:40110. doi: 10.1007/s11897-013-0155-7

4. Ward ZJ, Bleich SN, Cradock AL, Barrett JL, Giles CM, Flax C, et al. Projected U.S. state-level prevalence of adult obesity and severe obesity. $N$ Engl J Med. (2019) 381:2440-50. doi: 10.1056/NEJMsa1909301

5. Alpert MA. Obesity cardiomyopathy: pathophysiology and evolution of the clinical syndrome. Am J Med Sci. (2001) 321:225-36. doi: 10.1097/00000441-200104000-00003

6. Patel H, Madanieh R, Kosmas CE, Vatti SK, Vittorio TJ. Reversible cardiomyopathies. Clin Med Insights Cardiol. (2015) 9 (Suppl. 2):714. doi: 10.4137/CMC.S19703

7. Reddy YNV, Anantha-Narayanan M, Obokata M, Koepp KE, Erwin P, Carter RE, et al. Hemodynamic effects of weight loss in obesity: a systematic review and meta-analysis. JACC Heart Fail. (2019) 7:67887. doi: 10.1016/j.jchf.2019.04.019

8. Bozkurt B, Hershberger RE, Butler J, Grady KL, Heidenreich PA, Isler $\mathrm{ML}$, et al. ACC/AHA key data elements and definitions for heart failure: a report of the american college of cardiology/american heart association task force on clinical data standards (writing committee to develop clinical data standards for heart failure). Circ Cardiovasc Qual Outcomes. (2021) 14:e000102. doi: 10.1161/HCQ.0000000000000102

9. Alpert MA, Terry BE, Mulekar M, Cohen MV, Massey CV, Fan TM, et al. Cardiac morphology and left ventricular function in normotensive morbidly obese patients with and without congestive heart failure, and effect of weight loss. Am J Cardiol. (1997) 80:736-40. doi: 10.1016/S0002-9149(97)00505-5

10. Daniels LB, Clopton P, Bhalla V, Krishnaswamy P, Nowak RM, McCord J, et al. How obesity affects the cut-points for Btype natriuretic peptide in the diagnosis of acute heart failure. Results from the breathing not properly multinational study. Am Heart J. (2006) 151:999-1005. doi: 10.1016/j.ahj.2005.1 0.011 phenotypes. Masked clinical signs, symptoms and imaging limitations delay diagnosis. The cardiac changes due to severe obesity are predominantly "adaptive." Resilience in younger HFO may allow regression of cardiac abnormalities with 20$35 \%$ weight loss. Based on accumulating evidence, HFO is less a HFpEF phenotype and more a reversible cardiomyopathy with substantial clinical and health policy implications.

\section{AUTHOR CONTRIBUTIONS}

The author confirms being the sole contributor of this work and has approved it for publication.

\section{ACKNOWLEDGMENTS}

Office of Rural Health and Whole Health support to build novel self-inquiry group clinics for heart failure at The Harry S. Truman Memorial Veterans' Hospital.
11. Fitzgerald KN, Hodges R, Hanes D, Stack E, Cheishvili D, Szyf M, et al. Potential reversal of epigenetic age using a diet and lifestyle intervention: a pilot randomized clinical trial. Aging. (2021) 13:941932. doi: 10.18632/aging.202913

12. Scheffer M, Bolhuis JE, Borsboom D, Buchman TG, Gijzel SMW, Goulson D, et al. Quantifying resilience of humans and other animals. Proc Natl Acad Sci USA. (2018) 115:11883-90. doi: 10.1073/pnas.1810630115

13. Kitzman DW, Brubaker P, Morgan T, Haykowsky M, Hundley G, Kraus WE, et al. Effect of caloric restriction or aerobic exercise training on peak oxygen consumption and quality of life in obese older patients with heart failure with preserved ejection fraction: a randomized clinical trial. JAMA. (2016) 315:36-46. doi: 10.1001/jama.2015.17346

14. Chockalingam A, Dorairajan S, Anand K. Higher consciousness through selfinquiry can improve cardio metabolic outcomes, mental health, and resilience. Mo Med. (2021) 118:97-102.

15. Chockalingam A, Kumar S, Ferrer MS, Gajagowni S, Isaac M, Karuparthi P, et al. Siddha fasting in morbidly obese heart failure patients achieves significant weight loss reduces hospitalization. Explore. (2021). doi: 10.1016/j.explore.2021.12.003. [Epub ahead of print].

Conflict of Interest: Author AC was employed as an Advisor by the Cardiac Wellness Institute, Chennai, India. AC is co-founder of www.HiLifeJourney.org, a non-profit online self-help tool aimed at improving cardiac health and resilience through self-inquiry. AC is the author of Seeking HUNGER, a book encouraging self-inquiry for holistic health. The author declares that the research was conducted in the absence of any commercial or financial relationships that could be construed as a potential conflict of interest.

Publisher's Note: All claims expressed in this article are solely those of the authors and do not necessarily represent those of their affiliated organizations, or those of the publisher, the editors and the reviewers. Any product that may be evaluated in this article, or claim that may be made by its manufacturer, is not guaranteed or endorsed by the publisher.

Copyright (C) 2022 Chockalingam. This is an open-access article distributed under the terms of the Creative Commons Attribution License (CC BY). The use, distribution or reproduction in other forums is permitted, provided the original author(s) and the copyright owner(s) are credited and that the original publication in this journal is cited, in accordance with accepted academic practice. No use, distribution or reproduction is permitted which does not comply with these terms. 